\title{
How HCI Bridges Health and Design in Online Health Communities: A Systematic Review
}

\author{
Doğa Gatos \\ dcorlu14@ku.edu.tr \\ KUAR, Koç University, Turkey
}

\author{
Aslı Günay \\ asgunay@ku.edu.tr \\ Media and Visual Arts, Koç \\ University, Turkey
}

\author{
Güncel Kırlangıç \\ gkirlangic@ku.edu.tr \\ KUAR, Koç University, Turkey
}

\author{
Kemal Kuşçu \\ kkuscu@ku.edu.tr \\ Koç University, Turkey
}

\author{
Asım Evren Yantaç \\ eyantac@ku.edu.tr \\ KUAR, Koç University, Turkey
}

\begin{abstract}
This paper presents a systematic review of online health communities (OHCs) published between 2009 and 2020 in the ACM Digital Library. Aiming to consolidate the current issues, design knowledge, challenges, and tensions in OHCs, our analysis identified four high-level aspects related to the use and design of OHCs: (1) temporal: OHCs as transition spaces, (2) spatial: bridging experiential knowledge with medical expertise, (3) technological: exchanging and locating peer support, and (4) tension dimensions in OHCs. We further discuss methodological improvements and computing opportunities for OHC research and how to increase OHC members' agency in such a medically dominated context. These findings have the potential to inform future $\mathrm{OHC}$ designs and help researchers and designers position future contributions.
\end{abstract}

\section{CCS CONCEPTS}

- Human-centered computing $\rightarrow$ Interaction design process and methods; $\bullet$ Information systems;

\section{KEYWORDS}

online health communities, systematic review, social computing, information management, peer support, support seeking, transitions, ubiquitous health, digital health, health communication

ACM Reference Format:

Doğa Gatos, Aslı Günay, Güncel Kırlangıç, Kemal Kuşçu, and Asım Evren Yantaç. 2021. How HCI Bridges Health and Design in Online Health Communities: A Systematic Review. In Designing Interactive Systems Conference 2021 (DIS '21), fune 28-fuly 2, 2021, Virtual Event, USA. ACM, New York, NY, USA, 14 pages. https://doi.org/10.1145/3461778.3462100

\section{INTRODUCTION}

Seeking support is a key strategy to increase one's ability to manage health conditions, improve wellbeing, and adjust to life changes and challenges. Online health communities (OHCs) have become

Permission to make digital or hard copies of all or part of this work for personal or classroom use is granted without fee provided that copies are not made or distributed for profit or commercial advantage and that copies bear this notice and the full citation on the first page. Copyrights for components of this work owned by others than ACM must be honored. Abstracting with credit is permitted. To copy otherwise, or republish, to post on servers or to redistribute to lists, requires prior specific permission and/or a fee. Request permissions from permissions@acm.org.

DIS '21, fune 28-fuly 2, 2021, Virtual Event, USA

(C) 2021 Association for Computing Machinery.

ACM ISBN 978-1-4503-8476-6/21/06 . .\$15.00

https://doi.org/10.1145/3461778.3462100 crucial spaces for people suffering from various health conditions and their caregivers to find informational and emotional support around the world. According to a 2014 Eurobarometer report, 59\% of Europeans search online for health-related informational support [21]. Moreover, $23 \%$ of patients in the United States who have a chronic illness have gone online to seek others with similar health concerns [65]. In China, health websites are the most visited social service websites, with 100 million monthly users in 2011 [79].

OHCs allow people with varying health conditions to exchange knowledge and support with peers and experts in various online spaces such as discussion forums, social networking sites, and blogs. These communities help members to share and receive informational and emotional support without time constraints, geographic barriers [5], or financial cost. Besides logistical conveniences, OHCs have numerous advantages, including positive impacts on healthrelated behaviors, creating poly-vocality, and allowing people to anonymously share experiences and questions.

As an increasing number of people go online for health-related support, interest in the research on OHCs has peaked in the past decade. Previous literature reviews are valuable for illuminating granular aspects of $\mathrm{OHCs}$, including the effects of online peer-topeer interaction $[22,35]$ and participating in online health communities [74], behavior dynamics [13], participation styles [12], the empirical study of participation [43], and the social features of online health communities [37]. However, these studies largely focus on the behaviors of $\mathrm{OHC}$ members but neglect to report human-centered design issues. On the other hand, human-centered and computational ways to accommodate the exchange of support has gained interest in the field of Human-Computer Interaction (HCI), but the diversity and variance in topics pose challenges for researchers and designers in addressing gaps and mapping their work within this expansive research space. Besides, health-related experiences are also dominated by medical discourse in many contexts, presenting a design challenge to weave expert and peer support into OHCs. Thus, we see the importance in systematically reviewing the research on OHCs in a wider and more heterogeneous spectrum in order to map the conceptual and methodological issues in the field. To this end, we ask the following research questions: What are the current methods and challenges discussed in research on OHCs? What are the strategies to accommodate exchange of support related to health on online communities? How does HCI bridge health related experiences and design in $\mathrm{OHCs}$ ? 
To address these research questions, we conducted a systematic review of publications on online communities related to health and caregiving in ACM Digital Library, which contains a comprehensive HCI archive. The aim was to create a developed overview that can serve as a starting point when researching and designing online health communities for patients suffering from different conditions and battling life challenges as well as their caregivers. That being said, our aim is neither to provide an ultimate solution to the issues in $\mathrm{OHC}$ research, nor discuss each issue in detail. This review can rather be read as a roadmap into the $\mathrm{OHC}$ literature in the filed of HCI.

With this systematic review, we make the following contributions to HCI: First, we presented a descriptive overview of the current research on OHCs through an analysis of publication year, targeted communities, methodologies, and types of support identified in the reviewed papers. Second, we consolidated the design knowledge that these publications produced regarding the temporal, spatial and technological aspects of, and tensions in OHC design. In the remainder of the paper, we first describe the systematic review method we followed to investigate the most state of the art on OHCs. We then present our findings in two parts. The first part overviews the existing $\mathrm{OHC}$ research, including publication year and venue, targeted communities, roles, research sites, utilized methodologies, and ethical considerations presented in the reviewed papers. The second part highlights the emergent design knowledge extracted from the reviewed papers that is collectively salient or contrasting. We conclude the paper after discussing the gaps and future avenues for $\mathrm{OHC}$ research.

\section{METHOD}

This study focuses on OHCs and systematically reviews the existing research on the matter. Systematic review is a method that identifies, selects, and evaluates existing studies and scholarly contributions based on a set of formulated research questions [19, 34]. To ensure quality in this review, we followed the PRISMA [62] systematic review checklist (see auxiliary material). We present our review method in detail below. Steps 1-3 report the data collection phase, whereas Step 4 explains how the data are analyzed.

\subsection{Step 1: Retrieving Relevant Publications}

Source selection: We chose the ACM Digital Library as our database, because it aggregates research across multiple disciplines and maintains significant coverage of the field of HCI. Search query: The search query (see Figure 1) used to find relevant publications is compiled after scanning the titles and abstracts of a sample of publications retrieved through an initial desktop search and discussions among all authors. Timeframe: We conducted the search in June 2020 and did not restrict the search to a specific timeframe. We included all papers published before June 10, 2020-the date of our search-and the oldest publication retrieved with the search query was from 1988. Result: This retrieval of relevant publications resulted in 491 papers, which comprised a range of publication formats as well as duplicates.

\subsection{Step 2: Identifying Eligible Publications}

The goal in this step was to identify papers eligible for reviewing. Accordingly, the retrieved papers were first filtered through the ACM Digital Library's Research Article filter and were then eliminated by one of the authors based on the following exclusion criteria:

- Publications written in a language other than English

- Publications that were not full, peer-reviewed papers (e.g. dissertations, demos, workshop calls, short papers)

- Publications already in press but not yet published (due to unknown prospective publication date)

- Duplicates (of studies and publications)

Result: This step resulted in 162 publications, narrowing the earliest year covered by the review to 2007 . All papers were imported to a local drive in preparation for the next round of elimination.

\subsection{Step 3: Thorough Elimination}

This time the goal was to select papers that focus on humancentered aspects of OHCs. To do so, eligible papers underwent more rigorous scrutiny to eliminate more papers based on the following exclusion criteria:

- Papers not informing the design of support spaces (through, for example, explicit design implications or suggestions for practitioners)

- Papers not reporting on a user study

Results: This step resulted in the exclusion of technical research that does not cover human factors and computational modeling proposals that lack human-centered studies. The final round of elimination resulted in a total of 36 papers for the analysis, narrowing the earliest year covered by the review to 2009. A list of the 36 publications used in this review can be found as auxiliary material.

\subsection{Step 4: Analysis}

The analysis comprised two steps. First, information such as research phase, research site, type of data, user roles, community types, types of support discussed, targeted health condition and life challenge were extracted from publications and entered into a database for further annotation and analysis. The two authors performed the extraction independently in order to ensure an unbiased analysis. Disagreements between the two authors' analyses were discussed with all authors and reached to a consensus to present the descriptive findings in Section 3.

Second, the results and discussions from the studies in the corpus were analyzed qualitatively (the first and second author analyzed all papers, and the other authors each analyzed eight papers). The authors collaborated to extract, enter into a database, and code the concerns and implications regarding the design of OHCs from the papers (coding). Following a thematic analysis approach [10] (see Figure 2), all the authors collaboratively sorted the coded data based on the emergent initial themes. They then revised these initial themes in two rounds of discussion and identified further commonalities which led to the final themes: (1) Temporal Aspects: OHCs as Transition Spaces, (2) Spatial Aspects: OHCs as a Bridge Between Experiential Knowledge and Medical Expertise 


\begin{tabular}{ll}
\hline TITLE & communit* OR group OR peer* OR collectiv* \\
\hline AND & \multicolumn{1}{c}{ AND } \\
ABSTRACT & $\begin{array}{l}\text { online OR digital OR "social media" OR "social network" } \\
\text { OR internet OR forum }\end{array}$ \\
\hline ABSTRACT & $\begin{array}{l}\text { health OR care* OR patient OR condition OR illness OR diagnos* } \\
\text { OR disorder OR medical OR “life change" OR "life transition" }\end{array}$ \\
\hline TEXT & support* OR seek* OR search* OR shar* \\
\hline
\end{tabular}

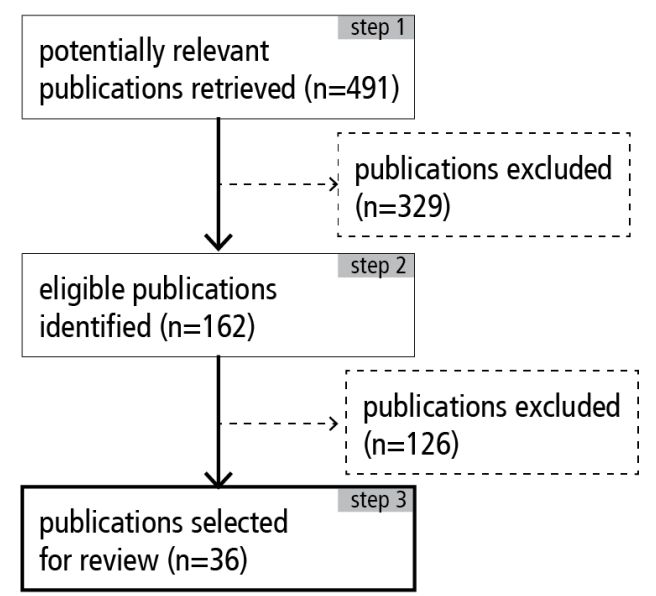

Figure 1: LEFT: Search query (AND between rows, OR between search terms) entered for retrieving potentially relevant publications. Asterix $\left(^{*}\right)$ is used after a keyword to find all words beginning with that root. RIGHT: Reviewing process.

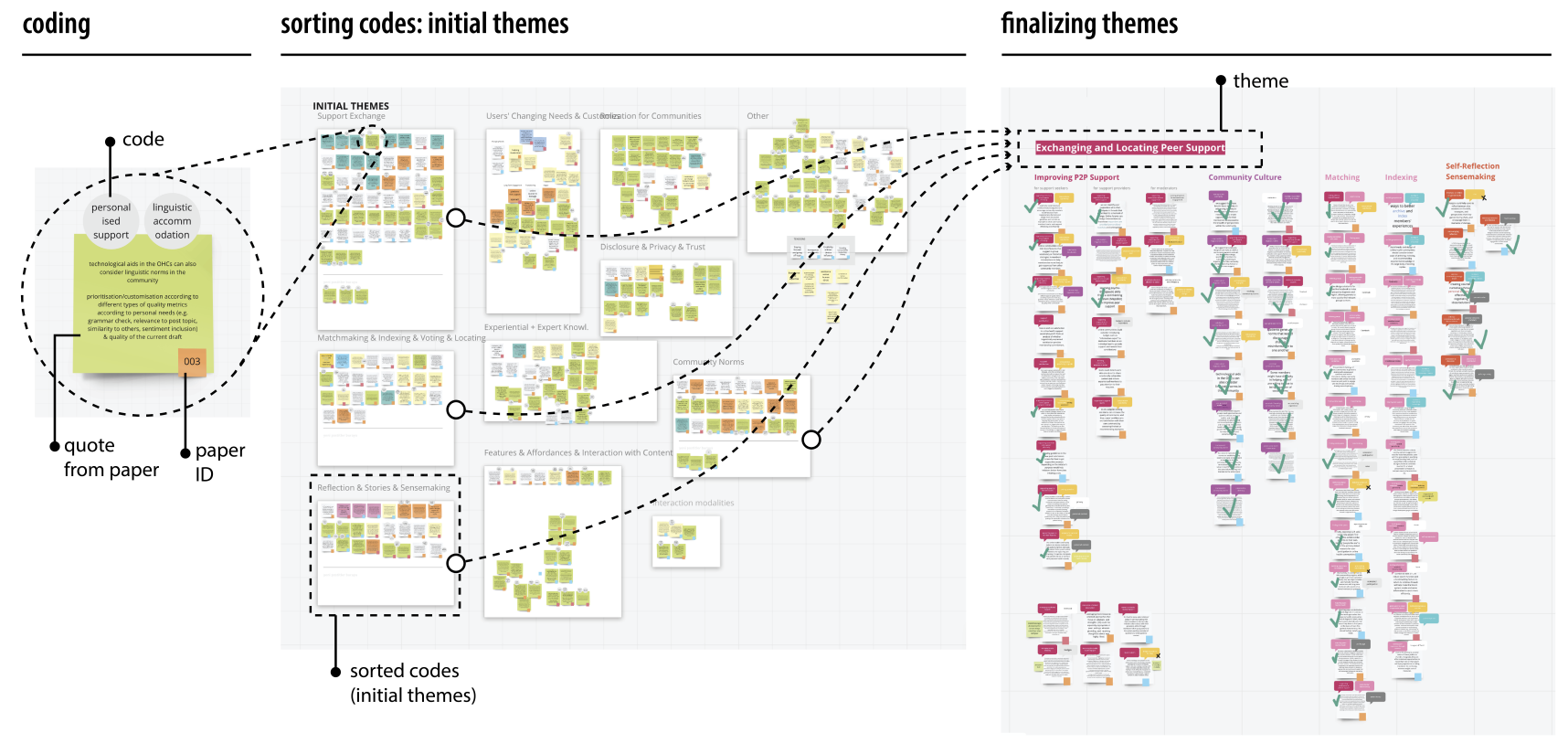

Figure 2: Thematic analysis process carried out on Miro.

(3) Technological Aspects: OHC Features for Exchanging and Locating Support, (4) Tension Dimensions in OHCs. We will explain these themes in Section 4 after we outline our descriptive findings regarding the $\mathrm{OHC}$ research in the literature in the following section.

\section{OVERVIEW OF OHC RESEARCH}

\subsection{Publication Years and Venues}

The number of studies on online support spaces for health and caregiving communities published in the ACM Digital Library trended upward over the years. Of the 36 papers, 27 were published in the past five years (2015-July 2020). Remarkably, no papers were published between 1988 and 2008 that met our selection criteria. Among all the publications that were reviewed (proceedings $n=34$, journal $\mathrm{n}=2$ ), $\mathrm{CHI}$ (Conference on Human Factors in Computing Systems; $\mathrm{n}=16$ ) and CSCW (Computer Supported Cooperative Work; $\mathrm{n}=11$ ) were the most represented conferences, followed by GROUP (ACM International Conference on Supporting Group Work; $n=4)$, DIS (Conference on Designing Interactive Systems; $n=1$ ), TOCHI (ACM Transactions on Computer-Human Interaction; $n=1$ ), Communication Design Quarterly ( $\mathrm{n}=1), \mathrm{C} \& \mathrm{~T}$ (International Conference on 
Communities and Technologies; $\mathrm{n}=1$ ), and WEBSCI (Annual ACM Web Science Conference; $n=1)^{1}$.

\subsection{Targeted Communities, Sites, User Roles and Types}

Almost all the papers $(\mathrm{n}=30)$ we reviewed targeted a single health condition (see Figure 3). Three papers studied multiple health conditions [29, 33, 46], and two papers reported on studies that examined communities of parents with neurodiverse children [1,83]-these were marked both as parenthood and special needs in the analysis. The most studied online communities were cancer $(\mathrm{n}=8)$, diabetes $(\mathrm{n}=7)$ and mental health $(\mathrm{n}=6)$ communities. Following these were eating disorders and weight loss $(\mathrm{n}=4)$, womanhood $(\mathrm{n}=4)$, substance use $(\mathrm{n}=4)$, special needs $(\mathrm{n}=3)$, general health $(\mathrm{n}=3)$, parenthood $(\mathrm{n}=2)$, dementia and Alzheimer's $(\mathrm{n}=2)$, and other conditions and life challenges, such as venous thromboembolism $(n=1)$, pain management $(n=1)$, and bereavement $(n=1)$ communities.

When we considered the kind of community sites that were investigated, we found that the online spaces specific to a health or caregiving community, such as the American Cancer Society [80] or CaringBridge [41], are the most frequently researched sites $(n=19)$. After these communities, the most common sites are existing social networking sites (Facebook, $n=5$; Instagram, $n=2$; and Reddit, $n=2$ ), which are not designed solely to be health communities yet have features that have been adopted as such. We also discovered four papers that investigated an interface element or component of a site, such as a chatbot [60] or a predictive model [63]. Seven papers did not report a study from a community site but instead conducted interviews with individuals who were members of possible future communities or existing ones that can inform the design of such sites (e.g. [11, 56]).

We also examined the framing of the roles of users in our set of papers as it pertained to their formal but not emergent or informal roles in communities. We found 34 papers that studied all the members of the scrutinized community without specifying the roles of users. Only a few papers (e.g., [38, 83]) mentioned that the community they studied involved different formal and informal roles, such as teachers and admins, but these studies did not focus on how these different roles affected their findings. Two papers studied moderators specifically [32, 52], whereas only one paper conducted a study with experts [33]. Our paper discusses user roles in detail, and we will present our relevant findings in the next section.

Lastly, we reviewed the types of support seekers regarding their relation to the person with a health condition. 15 papers did not specify whether they studied patients or their proxies, and generally referred to users as "community". 16 papers specifically investigated patients, who seek and share support for themselves (see "self" in Figure 3), whereas six papers focused on proxy seekers (users seeking support for another person with a health condition, e.g., caregivers and parents, surrogate seekers). One paper did not investigate the support seekers, and interviewed health experts regarding an $\mathrm{OHC}$ [33].

\footnotetext{
${ }^{1}$ We had eligible papers from other venues in ACM that are closely related to our research (e.g., DigitalHealth), but none met our review criteria and were eliminated in Step 3.
}

\subsection{Methods \& Ethical Considerations}

We evaluated the purpose of our study using the three main design phases as suggested in [18]: (1) Requirements where users' needs and requirements are elicited for designing a community, (2) Design, where ideation and prototyping took place, and (3) Evaluation, where the community or its components are evaluated for further development. Interestingly, papers reported previous studies largely in the requirements $(n=33)$ and evaluation $(n=5)$ phases, with only two papers in the design phase. The only use of generative methods we identified in the corpus was a design activity following an interview [57], but there was no mention of other methods such as design workshops or other co-design activities to design an $\mathrm{OHC}$.

We analyzed the research methods used and found that 19 papers reported a single data-collection method, such as interviews or digital ethnography, whereas 15 of the 36 papers reported mixedmethod studies. The methods used in our corpus of papers are as follows: (1) Utilizing user-generated $\mathrm{OHC}$ data is the most prominent method used to evaluate the OHCs and investigate the use and behavioral patterns of the users of existing OHCs. Here, researchers retrieve pre-existing content from $\mathrm{OHCs}$ and do not interact directly with users. A significant number of papers $(n=24)$ reported studies conducted with online user data (e.g. $[42,80])$. While few papers utilized user data in order to examine the qualitative aspects of a community through observation (e.g.,[38]) and digital ethnography [83], most papers analyzed user data quantitatively or to build computational models and generate algorithms (e.g.,[41]). The timeframe for data retrieval from communities varied; some papers used longitudinal data ([81]: 2003-2018), whereas some retrieved a month-long data [83]. (2) Interviews $(\mathrm{n}=20)$ were used to gain insights from individuals with a health condition or caregivers in order to inform the design of OHCs (e.g.,[1,11]). Interviews were generally combined with other methods such as surveys (e.g.,[67]) and observation (in the online community) (e.g.,[84]). Two papers $[33,60]$ reported studies that used user-generated data from OHCs for discussions with interview participants. (3) Surveys or questionnaires were used to understand individual experiences with OHCs (e.g. [69]) and to evaluate a developed OHC component (e.g., [60]). In addition to these methods, we identified usability studies $(\mathrm{n}=2,[29,57])$, focus groups $(\mathrm{n}=3,[52,72,73])$, expert reviews $(\mathrm{n}=1,[29])$, shadowing $(\mathrm{n}=1,[73])$, fieldwork $(\mathrm{n}=1,[72])$ and benchmarking $(n=1,[29])$. The identification of the methods used also provided a glimpse into the theories the papers grounded their work on. We saw a diverse set of theories and therapy models from sociology and psychology regarding the member roles and coordination in cooperative work (e.g., interactional and structural models in division of labor), collective sensemaking (e.g., communities of practice), agency (e.g., feminist theory), privacy and norms (e.g., selfdisclosure theory, speech accommodation theory, behavior change, cognitive structuring).

As health-related experiences may be sensitive and require additional considerations when conducting research on the matter, we considered how the papers we reviewed approached user privacy and safe conduct. We searched the corpus using the keywords "IRB, ethic-, approv-, consent, institut-, board, privacy". Apart from general procedures such as obtaining ethics approval from institutional boards, obtaining consent from participants, and removing 


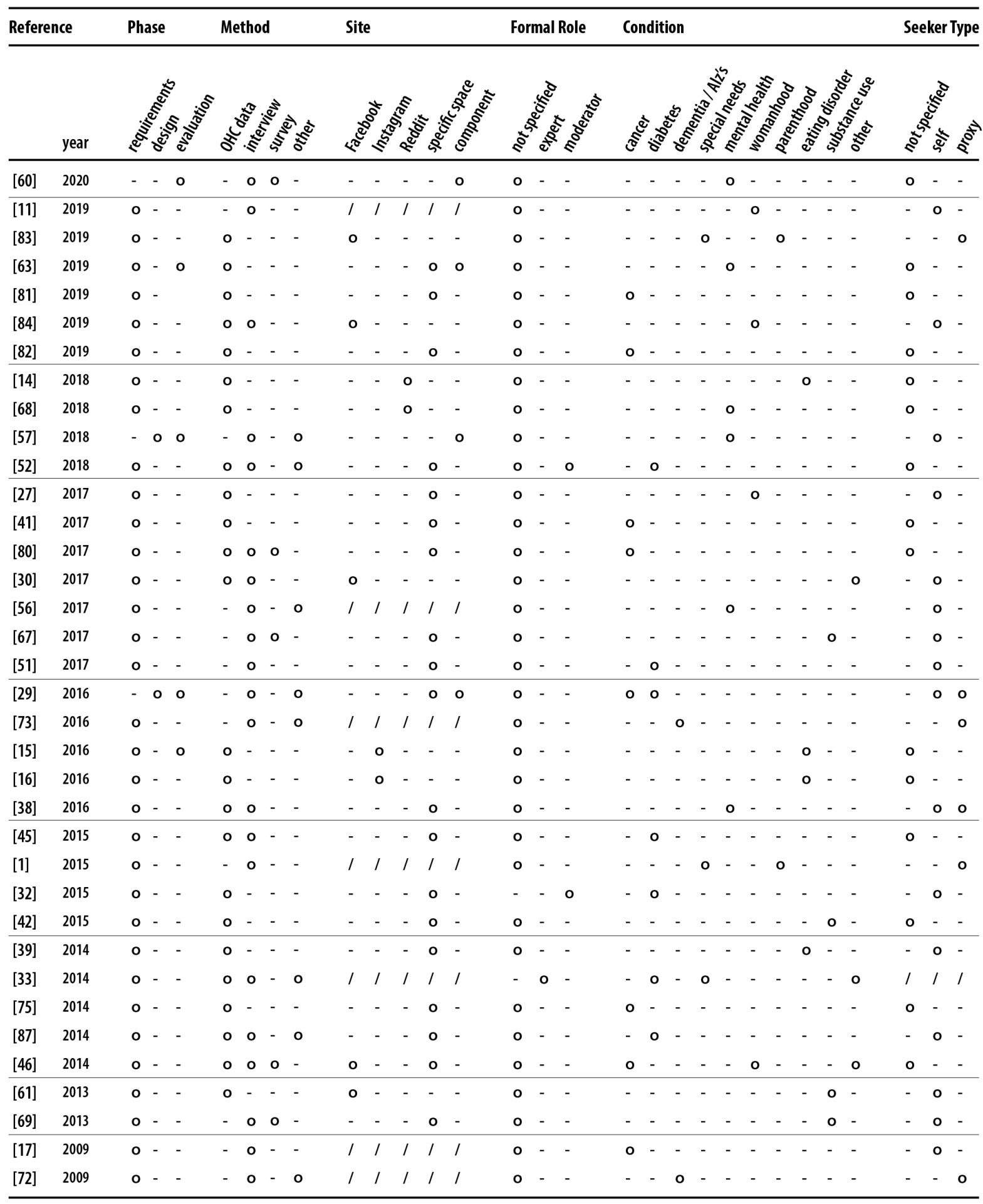

Figure 3: An overview of targeted research phases, methods, sites, OHC users' formal roles, health conditions and support seeker types identified in our corpus. -: not relevant; o: relevant; /: not applicable (e.g., no research site used for interviews). 
identifiable user information from quotes for publication, we identified the following strategies when utilizing user-generated data for $\mathrm{OHC}$ research: (1) procuring data anonymized by the community owners prior to the research [42], (2) creating a research account in the community [67, 87], (3) contacting administrators to enter and conduct research in groups [67, 83, 84, 87], (4) contacting members personally for permission to use their data [84], (5) giving community members the option for opting out from the research [30, 83, 84], (6) removing personal identifiers from the data before analysis [41, 60, 63, 68, 82], (7) applying machine-learning models for content analysis instead of human annotators [82], and (8) paraphrasing content for publication to conceal in search engine results $[68,82]$. For interviews and other methods, (1) risk assessment (evaluating the potential harm to oneself or others or the worsening of symptoms) [57] and (2) offering participants the opportunity to consult with counseling services [60] are used to ensure safe study conduct. Remarkably, 16 papers did not mention their ethical procedures [11, 14-17, 27, 39, 45, 46, 51, 52, 69, 72, 73, 75, 80].

\section{MAJOR THEMES IN OHCS}

After providing an overview of $\mathrm{OHC}$ research in the previous section, we now present the salient themes regarding the use and design of $\mathrm{OHCs}$ that emerged in the papers we reviewed. We organized these themes based on the four overarching aspects of $\mathrm{OHC}$ design and use: temporal, spatial and technological aspects, and the tensions that surface due to the complexity of OHC user needs, $\mathrm{OHC}$ research methodologies, and $\mathrm{OHC}$ design decisions.

\subsection{Temporal Aspects: OHCs as Transition Spaces}

This category uncovers the implications about temporal aspects of OHCs. In particular, these implications answer when and why users join, need, abandon OHCs, and how users' journeys and needs in OHCs change over time. In the following three subsections, we examine how OHC publications, generally, approach changing needs during health transitions and, specifically, address the diverse courses of individuals actions-such as members' roles and commitment-in OHCs due to changing needs.

4.1.1 Becoming a Patient and Transitioning. OHC studies contain a prevalent emphasis on the introduction of online communities to people's daily lives during a period of change in their or their loved ones' health conditions, making these online communities transition spaces [1, 11, 17, 30, 41, 46, 56, 61, 67, 73, 80-84, 87]. Life transitions are usually abrupt discontinuities within life experiences due to turning points, new beginnings, or endings [9]. Incurring a health issue is one of the multitudinous types of life disruptions; other examples include ending a relationship by a death or breakup or moving to a new city, college, or job [11,41, 46]. Although few of the publications we reviewed explicitly scrutinize the concept of transition (e.g.[11, 46]), many insinuate a relationship between transitions and $\mathrm{OHC}$ support, stating that $\mathrm{OHCs}$ are places people turn to when, for example, they are newly diagnosed, begin a healthcare journey, face crucial healthcare events, enter a new and unfamiliar reality, deal with changing life circumstances, or begin to be an informal caregiver. Regarding the spectrum of reviewed papers, health-related transitions may be unanticipated or planned and, relatedly, can be negative (e.g. cancer, neurodiversity, or Alzheimer's) or ostensibly more positive based on social norms (e.g. pregnancy, birth, parenting). They may fall more to the medical side of the health spectrum (e.g. diabetes, mental health) or on the wellness side (e.g. managing weight, gaining healthy eating habits, practicing mindfulness) and can be chronic or acute or, similarly, permanent or temporary. In any case, individuals undergoing such transitions need navigation skills and coping spaces, including support networks, because these are key mediating variables that influence the trajectory and emotional intensity of their transition [9].

4.1.2 Stages of the Condition and Changing Needs of Users. In line with the different phases a transition may comprise, such as encounter, sense-making, adoption, and resolution [9, 46], individuals participating in an online community may have evolving needs and expectations. We noticed an emerging stage-based approach to address individuals' needs in the publications we reviewed. Many publications highlight that health conditions usually involve multiple stages and levels and that those stages are highly interrelated with changing needs. The gestational stages of pregnancy [11, 27], Alzheimer's, Parkinson's disease, and renal failure [36, 40, 78] are some examples that include medically defined stages and these publications recommend structuring the OHCs according to these stages. However, it is important to note that health-related changes are not necessarily linear, even if the medical treatment of a condition is divided into specific stages. Accordingly, the publications also contain a pervasive expression of the importance of considering changing personal needs with different types, fluctuations, urgency, and prioritization in addition to the variety of needs different $\mathrm{OHC}$ members possess and the diverse types of support they seek and provide (e.g. emotional, informational, material). It is the general consensus that OHCs should engage people during highs and lows and in-the-moment states [38, 52, 56, 57] of their health journey. Also, they should be mindful to the criticality of the support requests $[56,68]$ by providing customizable and adaptive features, yet regarding the balance between competing priorities and the social fabric of communities [51,52].

4.1.3 Changing Roles and Interactions in OHCs. Many roles-doctors, nurses, interns, and other specialists and caregivers-exist in offline health settings and collaborate for the holistic care of patients. However, the objective in $\mathrm{OHCs}$ is not to mirror offline healthcare elements in online settings [33]. In a basic outlook, OHCs commonly have structurally defined and straightforward user roles [81], comprising registered members or visitors as well as moderators, organizers, and administrators [32, 33, 38, 60, 82, 87]. Many papers mention a shift in the roles and experiences of community members, from being an support seeker to a provider-mostly in tandem with entering the $\mathrm{OHC}$ as a newcomer/newbie and becoming a veteran/old-timer with prolonged experience in the health condition and community membership [1, 29, 32, 33, 38, 39, 41, $45,46,51,52,60,61,67,73,80-82,84,87]$. There is also a common, binary distinction between two groups of roles: lurkers (i.e. only-reading members, information seekers, inactive members) and active contributors (i.e. dubbed posters, regulars, core members) $[1,32,39,45,52,61,73,80,82,87]$. A look at the role dynamics based on behaviors reveals the emergent roles, such as welcomers, 
story sharers, all-around experts, private communicators, informational support seekers, private support providers, and knowledge promoters. Other group membership roles are addressed with consideration of the differences between tasks, socio-emotional, and individualistic roles, such as harmonizer and recognition seeker [44]. More special roles can also emerge depending on the specific community dynamics, examples of which are Shill and Quantified Self in a weight lost community [39]. [32] stated that labels for the roles can vary between communities, yet broader shared concepts around information and emotional support exist in many online forums. According to our review, even the names assigned to different roles imply their transience, not only according to "temporal" issues and "functions" but also to the related "motivation" and necessary "privacy" alterations.

A considerable number of OHC members, especially newcomers, are said to drop out before benefitting from or providing benefit to the community [80]. Membership retention may not necessarily be the result of community dynamics or the design of online spaces, but may be in relation to the members' changing life circumstances and needs to a certain extent [29,46]. Still, analyzing members' drop out or migration behaviors is important to inform the design of sustainable OHCs and foster smoother individual transitions between online and offline spaces as well as between different online platforms when needed [46]. We outlined three major reasons for discontinuity in participation: resolution and recovery, unmet or conflicting needs, and threats to wellbeing. First, the resolution of a support need and disease is a reason for drop-outs [30, 46, $80,81]$. When individuals become symptom-free, they may not need to participate in the community. Some could participate in an online platform merely to retrieve specific information-like a transactional resource-and decision-making [81], after which they have no sustained commitment. Likewise, individuals with short-term support needs or changing needs may drop out [80].

Second, when support seekers' needs are not met, they are reluctant to maintain their commitment. Newcomers in particular may make initial estimations about whether the community is worth their effort and time [80]. The quality of communication is crucial for the fulfillment of needs and the presence and quantity of communication [38, 46, 60, 80]. Apart from unanswered posts and questions, unpractical and unsupportive responses are likely to result in support seekers' disengagement [60]. Conversely, a support-provider's perception of being unable to satisfactorily provide support can result in fractured motivation and engagement due to a reduced sense of worthiness and competence. This sometimes results in withdrawal from the community or refraining from posting, especially when the support provider is a newcomer and is already in a psychologically more vulnerable condition. Moreover, the suitability of the community content and affordances (e.g. channels, norms, navigation and posting functions) to needs is important for satisfactory information retrieval, identity management, and communication. People approach and repurpose physical-and even social-affordances and constraints in OHCs such that they do not hinder their transition and better support their changing roles and needs. So, members sometimes drop out and migrate to other groups or sub-groups and even may launch new communities if the current one feels insufficient and not-targeted [1, 11, 46, 84].
Third, the papers highlighted instances of explicit and implicit-or direct and indirect-threats to community members' wellbeing and daily-life circumstances [11, 14, 38, 46, 56]. Implicit and indirect threats represent the circumstances when participating in a community and reading its contents is not beneficial due to exposure to displeasing or triggering content $[38,46]$, thus recalling or anticipating negative experiences, feeling emotional burdens, and exacerbating symptoms [56]. Outsiders or judgmental individuals may interpret expressions of self-disclosure through a normative lens. They may even use personal information against the poster, threatening personal boundaries and safety. However, papers that presenting such incidents are rare-one mother, for instance, was reported to child protective services because she was struggling with her baby [11]. Perceived risks for personal safety, though seldom, occur due to others' deliberately harmful messages, trolling, cyber-bullying, and harassment; this may even transpire at a larger scale when a community is under attack $[14,56]$. Many of these incidents are discussed in relation to the significance of assuring anonymity, data protection, and the exclusion of third-parties in order to preserve safety and trust.

\subsection{Spatial Aspects: OHCs as a Bridge Between Experiential Knowledge and Medical Expertise}

In this part we report on the implications that are concerned with how OHCs connect online and offline aspects of members' health journeys, and how OHCs are positioned in medical domains as well as social and cultural contexts.

4.2.1 Cultural and Public Understanding of Conditions. The offline transformative power of OHCs beyond the online community is usually situated in our paper set. Health-related experiences are not only medical but are situated along social, cultural, economic, and political contexts, sometimes triggering conflicts between patients, their inner circles, and society due to the lived experiences of patients and what others perceive, label, and expect due to norms (e.g. pregnant woman conception, in [11]). Several papers focus specifically on how culture molds the interpretation of symptoms, treatments, behaviors, and social interactions (e.g. [38, 63, 87]). An illustrative example appeared in [38], which noted that Chinese cultural beliefs value collectivistic relationships and the power of inner self-control. Consequently, people with depression tend to hide their thoughts and feelings, afraid to be perceived as incapable of resolving inner conflicts and having low morality. They would lose their jobs and suffer discrimination even within their social circle. Traditional Chinese medicine emphasizes the integrated body-mind relation and promotes the resolution of mental problems with the treatment of somatic issues. This results in Chinese people describing somatic symptoms to explain their mental problems rather than reflecting on and disclosing their thoughts and feelings. Yet OHCs help such patients self-disclose, find help, and collectively raise awareness about culturally affected conditions and their treatment [38]. Likewise, another study on diabetes reported that OHCs help Chinese patients coordinate their treatment choices, routine management, and social participation, because social eating and drinking and table culture are important elements in 
organizational and social life, posing challenges to individuals with diabetes [87]. Overall, OHCs empower individuals and communities to challenge existing norms by individually and collectively critiquing, reflexively re-crafting, and increasing awareness outside the OHCs in the general public and among policy and lawmakers [1, 11, 30, 38, 68, 73, 83, 87]. Furthermore, most reviewed papers articulate civic changes and resource mobilization, extending transformations in understanding as a result of empowerment and advocacy through OHCs $[1,11,30,32,83]$.

4.2.2 Intertwined Nature of Daily and Medical Expertise. Not all $\mathrm{OHCs}$ involve healthcare professionals, but all the reviewed $\mathrm{OHC}$ papers encompass informal experiential and formal medical knowledge embedded in the day-to-day experiences of patients and their caregivers. Some papers touch upon the intentional integration of medical expertise (e.g. [27, 30, 32, 63, 83, 84]), and expressed the importance of volunteer professionals [38] for the effective moderation of an OHC. Papers articulated the importance of OHCs for the integration of daily expertise beyond medical knowledge. Medical expertise and patients' own expertise, in the form of daily experience around health management, are innately intertwined [33]. Though recognizing the significance of medical knowledge, OHCs should not be perceived as an alternative for medical expertise [32], and the multifaceted aspects of health-related daily experiences should be considered beyond medical discourse [83]. Content about daily experience in OHCs involves narratives about navigating daily life around a health issue, emotional reactions, intimate details, and thoughts (e.g. [1, 27, 29, 30, 41, 52, 80, 82, 84]). They enable patients to facilitate self-disclosures, even of stigmatized experiences $[68,81]$, ask uncomfortable and strange questions [11], learn about daily and local events and places related to a health issue (e.g. where to find wigs, petting zoos, children's museums, playgrounds that are accessible for children with special needs) [33, 83], vent about daily experiences [11], contextualize and validate personal experiences with both similar and diversified examples from others $[27,73,82]$, and empower individuals as informed patients and families armed with an arsenal of coping strategies [27, 32].

4.2.3 Guidance for Receiving Offline Medical Expertise. OHCs provide an online space for attaining a certain extent of clinical knowledge. They do this either directly through the integration of medical expertise, as in the form of healthcare members or moderators, or indirectly through medical information embedded in the peer patients' lived experiences. Many publications touch upon the importance of online spaces for guiding individuals while receiving offline medical expertise. We observed three major points of guidance in $\mathrm{OHCs}$ for doctor visits: before, during, and after consulting offline medical expertise.

First, OHCs usually advise support-seekers to see their healthcare providers [27, 30, 32, 33, 39]. This is especially important due to the sensitivity and immediacy of health-related issues and liability concerns. Once an individual is determined to see a doctor, OHCs are informative about locating and selecting medical expertise, offering pointed, quality, and skilled healthcare services and professionals [11, 30, 32, 33, 83, 84]. More specifically, OHCs inform individuals about how to choose doctors, what types of doctors to see based on specialty, who to choose or avoid according to doctors' manners and reputations, and where to find them. Second, we saw a recurring emphasis on the role of OHCs in offline doctor visits, guiding patients to have good doctor-patient communication $[1,15,27,30,32,33,83,84]$. Examples about the significance of online guidance for offline doctor-patient communication include devising strategies about how to talk with healthcare providers and providing advice about communication skills medical professionals will take seriously [32, 33, 84], accurately describing a sensation and its location [84], steering providers for a more specific diagnosis and treatment based on the current knowledge gained from OHCs $[1,84]$, and making the necessary preparations for appointments, such as specific documents or lists of questions, records of symptom type, frequency, and intensity [32,33,84]. The quality of patients' encounter with doctors is pertinent not only to physical and intellectual preparations through an informed and equipped presence at doctor visits but also to psychological preparations. OHCs can also help shape patients' expectations from encounters with physicians [30, 46, 69]. Third, OHCs also come into play after individuals begin viewing their own providers as individuals and wish to assess their doctor visits, receive second opinions about diagnoses and recommendations [27, 32], and sometimes ask whether to seek out another doctor [30,32].

Overall, specific design strategies include the incorporation of checklists, templates, forms, diaries, and self-tracking technologies to guide patients in preparation for and communication with their healthcare providers. Documents and diaries can be filled automatically based on patients' history and integration with personal health records and self-tracking technologies (e.g. [33, 84]). Though not directly related to OHCs' guidance for receiving offline medical expertise but certainly related to the incorporation of offline medical expertise, suggestions about collaboration with clinics are also noteworthy in order to examine and better utilize the content patients share [15] and to engage patients holistically from prevention to after-care [33].

\subsection{Technological Aspects: OHC Features for Exchanging and Locating Support}

Receiving and sharing support is the key attribute of OHCs. Therefore, we analyzed what design strategies and features were offered to exchange and locate support as well as the types of support the papers considered. Our review found a heavy emphasis on forum-style (posts and comments) public communication among community members for the exchange of support in OHCs. In general, dedicated $\mathrm{OHC}$ websites and social media platforms-such as groups on Facebook-have a public discussion board or forum as well as options for chat and email (e.g. [52, 82]) for private communication. Besides discussion boards and chat modules, we found other support exchange modes in our review, including video-supported communication, expressive writing, blogging, and assisted chat. We also found important strategies that were investigated to improve the exchanging and locating of support, which we will explain after clarifying the types of support investigated in our paper set.

4.3.1 Types of Support. Social, informational, and emotional support are the most common functional denominators of the diverse range of $\mathrm{OHCs}$ in the reviewed papers, as they were also visible in many other work related to OHCs [7, 53, 64, 76, 77, 85, 86]. This systematic review has posited that spiritual, financial, occupational, 
and civic support can also be substantial in OHCs even though these do not demonstrate a recurring pattern in all the papers. These coincide well both with the actual definition of health, which highlights a complete state of wellbeing, expanding beyond the mere absence of disease or infirmity [20,59], and with almost all holistic wellness dimensions (e.g., physical, emotional, intellectual/informational, financial, occupational, social, and environmental [2, 48, 58]). All support types have a mutual impact one another, just as all wellness dimensions are interconnected $[2,6,55]$. Although the health conditions addressed in $\mathrm{OHCs}$ vary in our review, the direct and indirect impacts of the informational support on the psychological states of the information seeker are the most obvious, and are even more apparent in communities that are psychologically more vulnerable (e.g. mental health support communities [60]). Another example is the occupational impact of emotional and financial support. When the careers of individuals facing a health-related transition-and of informal caregivers-are under threat, emotional and financial support can provide encouragement and empowerment in multiple forms (e.g. fund-raising and donation to support vocational decisions, collective re-negotiation of the social norms related to the career of new mothers [11]). Such support types also entail civic support, where individuals gain awareness about or access to basic human and legal rights in relation to their health condition, work, and relationships. Examples of civic support include fundraising to cover lawyer's fee [11], petition campaigns to reduce financial burden and effect political change [83], and posting about legal and policy issues, and embracing advocacy [1].

4.3.2 Linguistic Assistance and Norm Moderation. As most of the support exchange occurs verbally due to OHCs' affordances, many publications suggest ways to linguistically assist members when asking for or providing support. Linguistic assistance targets supportseekers to compose content that illustrates their health condition or history and helps them get responses. Proposed linguistic assistance features include tutorials for effective support-seeking [82], guidelines to better formulate questions to get supportive answers [32], rhetorical strategies to write content more likely to get responses from others [82], quality metrics [60] based on personal needs (e.g. grammar checks, relevance to post topic, similarity to others, including personal sentiments), warnings for under- or overstated experiences [33], prompts for including sources or links in the composed content [33], and probing questions and templates to help support-seekers articulate their history related to their health conditions [33]. Linguistic assistance is also suggested for support-providers in the form of personalized tutorials or training to provide better support [63] and commenting assistance that teaches psycho-therapeutic skills [60].

Besides improving the quality of the content created to seek or provide support, linguistic assistance in OHCs may be needed to conform to community norms and culture. Forming a community culture starts by creating norms, a typically user-generated set of rules and traditions that are shaped around a community's linguistic practices. These norms are crucial for conformity, unity, and acceptance by other members [68]. However, OHCs' linguistic norms are in some cases uncertain, especially for new members, and this uncertainty prevents new members from supporting other members, even if they wish to do so $[60,81]$. This implies the need to make linguistic norms as visible and transparent as possible to ensure community reach $[67,68]$.

One way to conform to community culture is moderation, where some members are encouraged to offer supportive, positive responses to posts while maintaining order through various control mechanisms [61]. The control mechanism here can be moderators, who have the power to ban members and censor content when necessary. However, the studies implied the inadequacy of individual efforts to scan content, and thus highlights the need for assistance to form model support in the community $[14,60,61,67,68]$ other than moderators. Another approach is promoting peer moderation, which is already established in some communities by discouraging the spread of misinformation [33] and teaching members community norms [67]. The papers reiterate that paying attention to the emergent and changing roles of $\mathrm{OHC}$ members could also be valuable for intervention and norm moderation in OHCs [17, 33, 61, 81]. Some researchers discuss the incorporation of badges representing the roles of individuals as a specific role-based design intervention $[29,52,81,82]$. Badges can be informative to locate and provide support and, thus, increase trust in the retrieved support. Additionally, the incorporating badges in user profiles can motivate members for their contributions. Nevertheless, role-based interventions should not conflict with a nurturing community environment by highlighting competition, inequalities, and power imbalances $[51,52]$.

4.3.3 Indexing Support and Matchmaking. Another crucial aspect of OHCs for exchanging support is the ability to find similar peers and experiences [24]. Interestingly, publications elaborated on how to match peers and emphasized the importance of considering personal and granular similarities between peers that are beyond the shared health diagnosis. These similarities include shared experiences [51], common interests [32], patterns related to health condition [42], stages of the diagnosis [1], location or region [1], community roles (e.g. matching newcomers with welcomers) [42, 81], and sharing similar caregiving situations [73]. According to O’Leary et al. shared characteristics gain more importance especially in mental health contexts: "In contrast to dominant approaches that organize health communities around diagnostic labels, many participants desired tools that would help them to find peers on the basis of more fine-grained characteristics, like shared feelings, beliefs, and needs."[56, p. 1479].

We spotted two categories of strategy to encourage members to find and interact with peers. The first category suggests the automatization of matchmaking: directing peers' attention to the necessary threads [32,63], automatically matching newcomers with long-term members [42], and detecting shared interest [51]. The second category focuses on enhancing members' profile pages [1, 73], by including information such as member's expertise regarding the health condition [81], emotional support needs [81], region [1], stage and history of the diagnosis (patient history) [1], join date [1], and past shared content [1]. This category also entails a dedicated search for members' profiles [73] that are enhanced by highly granulated information. Although automatically matching users based on their needs and formal or informal roles in the community is an explicitly investigated topic, the use of enhanced user profiles as well as incorporating journeys and histories related 
to members' health conditions into community platforms surface as a need that warrants further study. Support-seekers' posts usually lack the necessary information [33], and peers and experts require more details about their history and context to be able to provide better, more personalized support. However, many existing OHCs or repurposed social media platforms are unable to display or offer spaces for support-seekers to provide such information. To overcome this mismatch between what support-seekers show and what support-providers need for the exchange of support, researchers suggest using mechanisms to produce probing questions for support-seekers to help them better explain their backgrounds and enrich their profiles [33]. These profiles may be enhanced to work as personal blogs [41] rather than merely displaying basic information. Using seekers' past contributions, posts, and comments and integrating them with support-seeking posts may also add further detail to their histories [33]. Mechanisms to facilitate matchmaking can, in turn, facilitate sensemaking both individually and collectively. Studies argue that the individual level of sensemaking is helpful for negotiation between members via personal stories [83] or shared artifacts or discussion tools [45]. Encouraging the practices of self-reflection [41, 57] and self-tracking [84] within the design spaces of OHCs were also claimed to be beneficial on the individual level, both intrinsically and extrinsically. The actions taken at the individual level would then become useful in the creation of collective sensemaking $[45,83]$.

In addition to matching peers based on their roles, needs and profiles, it is important to index the content on OHCs. As we explained earlier, individuals experiencing a new health condition or life transition turn to online communities to gain the experiential knowledge of their peers [17] which is shared in peer-to-peer interactions conversationally. These conversations encapsulate useful information and collectively create bases of knowledge. However, given the volume of members and the large body of potentially helpful content that is shared, helping users navigate and find existing yet fragmented information within that knowledge base becomes a challenge for OHC designers. Civan et al. [17], one of the earliest works in our paper set, explicitly investigate the locating and indexing of members' experiences on a breast cancer community platform and suggest problem-specific workspaces. Those workspaces are basically the collection of content that is organized around common issues about which community members frequently require and exchange support. They further suggest that members can organize the workspace by revising, extending, and annotating the content shared by others in order to facilitate the use of important or useful information. Another crowdsourced strategy to index the presented content is the use of folksonomy [73]. Folksonomy is a user-based classification method by annotating or assigning tags to content. User-assigned tags can accumulate tips, shared advice, and aggregate knowledge in the community while preserving "the conversational context in which the knowledge was expressed" [73, p. 3554]. In addition to these suggestions for indexing user content, researchers also highlight the aggregation of stories through crosslinks [38], automatically summarizing the given advice [17], and separating user stories with forum entries so stories do not get buried by entries [39].

\subsection{Tension Dimensions in OHCs}

While integrating algorithms, models, and assistance into OHCs is useful and vital to improve peer-to-peer support, the organization of content, and sensemaking, it accompanies several drawbacks and points of tension. Besides the dimensions we present below, a detailed discussion on the tensions such as staying focused and going off-topic, credibility without undue influence, norms versus inclusion, immediacy versus awareness in $\mathrm{OHCs}$ can be found in [67] and [51].

4.4.1 Sharing personal information vs. protecting privacy: The choice between disclosing information and maintaining privacy [51] or anonymity [67] is a major tension that surface in OHCs. OHC members may not be willing to provide detailed personal information, however this is needed to be matched with other members and to receive their support. Similarly, members, who are willing to provide support, need to contextualize the support seeker's condition and experiences to be able to give advice or help. Moreover, personal information may help building closer relationships between members [51], thus richer profiles are often needed for meaningful support. On the other hand, more and more research strives create awareness on the potential harms of disclosure of personal information online [3]. Although not discussed in detail in our corpus, this tension is even more crucial in the case of caregivers and parents of children with special needs $[1,83]$. OHCs provide important consultation to parents, particularly where state-based health services for individuals with special needs are not efficient. However, in this case, parents (or caregivers) disclose information online about the individual (mostly a child) under their care to seek support. Such displays expose children to other people without their consent and pose the risk of potential future discrimination $[8,70]$. As suggested by Ringland et al., designers and researchers should contemplate how to help parents become aware of the ramifications of seeking support online and acting upon it-alignment between parents in how to safeguard each other's children [66].

4.4.2 Optimized experience vs. freedom: To sustain communities, designers should consider ways to optimize the user experience. Matching members based on their emergent roles, such as matching newcomers with welcomers, and information experts with support seekers [81] can be a useful strategy to achieve this goal. However, such system-triggered matchmaking can also potentially "become a self-fulfilling prophecy, shepherding users into a particular activity path without giving them the full breadth of opportunity to explore other roles." [81, p. 12]. Similarly, monitoring and warning mechanisms, such as the detection of previously discussed topics or the censoring of depressing or inappropriate content may help communities prevent duplicate content and prevent exposure to unwanted content. Conversely, these mechanisms may also deprive members of their freedom of speech, expression, and discovery [38, 51, 52].

4.4.3 Similarity vs. diversity: Another tension that was articulated by $\mathrm{OHC}$ members and discussed in the papers was the duality of like-mindedness and diversity in OHCs. While OHC members like to hear from others who share similarities with them, they also want to be exposed to diverse perspectives [67]. Diversity may be considered as a core value of OHCs that have a large volume of members, but the multiplicity of the opinions can be overwhelming 
for members at the same time [51]. Given the abundance of approaches to locate and match members with similarities, its impact on promoting diversity in $\mathrm{OHCs}$ is needed to be further investigated.

4.4.4 Replicability vs. ethics: Use of user-generated OHC data generates methodological dilemmas, which has been of interest of HCI researchers recently. While it is crucial to ensure the protection of users' rights, the techniques used for user data de-identification are far from perfect [54]. Moreover, using open data facilitates replicability and is sometimes mandatory for submitting research papers. Under these circumstances, researchers should think of a "reasonable compromise between the priorities of ethical protection and of replicable science." [41, p. 73:6].

\section{DISCUSSION AND CONCLUSION}

The aim of this paper was to present an overview of research on OHCs and a consolidated account of design knowledge produced by $\mathrm{OHC}$ research in the field of HCI. Previous work on OHCs intensively focused on users' health behaviors without making a clear relationship between what those behaviors may mean for $\mathrm{OHC}$ design. Thus, we purposefully reviewed papers that elicited user insights, needs, behaviors, and expectations, and that have the potential to inform the design of OHCs in a human-centered way beyond tackling only the technical aspects of design, or reporting only the health behaviors of users without a design lens.

The analysis of the 36 systematically selected and reviewed papers on OHCs reveals three overarching frames: temporal, spatial, and technological aspects, as well as tension points between them which characterize OHCs and should be considered in OHC research and design. The temporal aspects put forward the importance of OHCs in facilitating individuals' transitions not only into becoming patients but also throughout a healthcare or caregiving journey. A stage-based approach draws attention; yet, there is an emphasis that changing needs and roles in OHCs may not be necessarily linear. Patients and caregivers use and repurpose physical and social affordances to facilitate their journey both within daily life and in OHCs. The spatial and contextual aspects of OHCs gain importance as OHCs bridge the experiential knowledge with medical expertise, and the offline contexts with the online, by playing a steering role in the offline that has a transformative power beyond the self and in multiple domains of life. More specifically, OHCs empower individuals and communities to challenge existing norms through their journeys by individually and collectively critiquing, reflexively re-crafting, and increasing awareness outside the OHCs in the general public and among policy and lawmakers. Moreover, OHCs not only provide informational and emotional support, but also more granular types of support such as spiritual, occupational, instrumental and civic empowerment. The findings also support and extend discussions in the literature about the importance of providing information about legal rights on digital health applications and forums [28]. Community members naturally develop ways of providing such a wide support range, even if the online platform does not offer relevant functions and mechanisms. These ways usually emerge in the form of shared content, tone of communication and collective actions upon an expressed need (e.g., fundraising), implying that the more intentional incorporation of holistic support mechanisms in the design of OHCs can contribute extra value in these online communities.

As per tangible design attributes and technological aspects, we see efforts in linguistic assistance and norm moderation in order to enhance the quality of the content, social fabric, and supportive culture of the online communities. Matchmaking and indexing appear to be crucial for locating and exchanging support, which could be based not only on shared medical characteristics, but on personal interests and changing needs and roles. We encountered an emphasis on sharing narratives and making stories easier to capture and find in order to claim medical expertise as patients and to provide expertise as experts when such actions were needed. This emphasis on sharing narratives harmonizes with the current narrative turn in medical domains [25], which are currently pursuing inquiries into personal experiences related to health conditions with a narrative lens. While narrating stories help individuals make sense of their own condition, it also facilitates receiving better, more individualized support from peers and experts by contextualizing one's own health condition-core features of OHCs. Future studies could consider mechanisms to better capture and index users' small and big stories $[4,23,26]$ by, for example, incorporating probing questions into personal health technologies. Generative methods, such as co-design activities have the potential to inform these mechanisms with the input of patients and experts, which may help extracting the topics necessary for the crowdsourced tagging of OHC content, as well as facilitating the discussion on individuals' agency in medical domain.

Relatedly, our analysis also revealed several methodological gaps in $\mathrm{OHC}$ research. First, although there was abundant explorative and evaluative research in our paper set, we did not spot any generative methods used for OHC design and research. This suggests that the involvement of users in the design of OHCs is likely to be lacking. We believe that the insufficient direct participation of users in OHC design hinders the creation of better ways to incorporate the experiential knowledge and agency of users into OHC structures. Second, online communities are often investigated in terms of users' emergent and informal roles, such as newcomers, story sharers, and knowledge promoters, but less frequently in terms of more formal roles such as admins and experts-a variety of professionals from related health domains. Members with formal roles undertake an important part in sustaining their communities by moderating content, gatekeeping, and promoting useful and accurate information. Although recent tendencies are to replace these roles with crowdsourced or automatized moderation, designers should consider how to bring together users with different formal roles, particularly support seeking members and experts, in order to craft and moderate content for the community. This collaboration could help participants reconsider how health-related information is produced and shared, a field typically dominated by medical and normative discourse.

Another shortcoming we found relates to ethical procedures, which were unclearly deployed in almost half of the publications we reviewed. Despite the numerous potential advantages of using social media in research-such as reaching large number of participants [49], reducing costs for large-scale research [50], studying longitudinal user interactions [31], reduced bias between researchers 
and participants [47]-the potential implications for privacy, confidentiality, and ownership [71] are not negligible. Not only do we suggest the explicit reporting of ethical procedures beyond IRB approvals, but also discussing the potential privacy implications that may emerge in the studies. Detailed reports and discussions on the subject matter can help future studies adopt ethical methods and inform the development of guidelines to preserve community and individual privacy while not undermining replicability. On the other hand, practitioners could think of ways to make $\mathrm{OHC}$ research beneficial for $\mathrm{OHC}$ members by integrating novel ethical and research dissemination structures within OHCs.

While design knowledge produced in the corpus was extensive, it showed the complexity of how OHC members' priorities, needs and expectations may diverge, how specific discourses and practices in health domain may affect individuals' use of OHCs, and how the social fabric of OHCs is prone to be disrupted by computing interventions. This complex picture indicates challenges for $\mathrm{OHC}$ researchers, designers and development of technical solutions; but also presents a reminder for future studies to pursue a balance between providing users automatization and freedom, means of support exchange and privacy, norms and inclusion, and similar experiences and diversity.

This paper makes a twofold contribution to the field of HCI: a descriptive overview of the human-centered research on OHCs, and a synthesis of spatial, temporal, technological aspects of, and dilemmas in OHC research and design. These contributions can serve future researchers and practitioners as a roadmap into the related literature as well as help them situate their work within this literature. We also provide the following directions for future research on $\mathrm{OHCs}$ based on this systematic review:

- Developing ways to incorporate holistic support mechanisms in the design of OHCs,

- Developing ways to better capture, share and index members' narratives, stories and journeys to support sense-making and help receive more granular support from peers and experts,

- Collaborative design with patients and experts to integrate experiential knowledge within a medical domain,

- Reporting the ethical considerations more transparently to help the development of safer research conduct in OHCs.

\subsection{Limitations}

An important limitation of this systematic review is that we retrieved papers only from the ACM Digital Library and, thus, may have missed relevant papers published in other databases and venues such as Web of Science, Scopus, PubMed and JMIR. Despite this limitation, this systematic review has a large coverage of publications that provide useful insights for the HCI field. It also has the potential to expedite future research and practice with its synthesis of studies that inform the design of OHCs through user needs, and with its review method which can be adapted for extending the coverage from other databases. A second limitation is that, this study purposefully reviewed papers that include a user study, explicit design implications, and suggestions in order to inform design and research with OHCs. We may have overlooked other studies that do not conform to these criteria but that provide useful viewpoints and insightful discussions about the development of
OHCs. Likewise, the review may not encompass a full range of the types of OHCs that we may not have captured in the HCI literature and with the explicit search criteria. And certain $\mathrm{OHCs}$ may have further idiosyncrasies that exhibit trade-offs with the examples in these papers. Lastly, our analysis concentrated largely on the findings and discussions of these publications, which may have neglected the lessons derived from the development of computing models and algorithms.

\section{REFERENCES}

[1] Tawfiq Ammari and Sarita Schoenebeck. 2015. Networked Empowerment on Facebook Groups for Parents of Children with Special Needs. In Proceedings of the 33rd Annual ACM Conference on Human Factors in Computing Systems (Seoul, Republic of Korea) (CHI '15). Association for Computing Machinery, New York, NY, USA, 2805-2814. https://doi.org/10.1145/2702123.2702324

[2] David J. Anspaugh, Michael H. Hamrick, and Frank D. Rosato. 2011. Wellness: Concepts and Applications (8th ed.). McGraw-Hill Education, New York City, NY, USA.

[3] Marco Balduzzi, Christian Platzer, Thorsten Holz, Engin Kirda, Davide Balzarotti, and Christopher Kruegel. 2010. Abusing social networks for automated user profiling. In International Workshop on Recent Advances in Intrusion Detection. Springer, 422-441.

[4] Michael Bamberg. 2006. Stories: Big or small: Why do we care? Narrative inquiry 16, 1 (2006), 139-147.

[5] Azy Barak, Meyran Boniel-Nissim, and John Suler. 2008. Fostering empowerment in online support groups. Computers in human behavior 24, 5 (2008), 1867-1883.

[6] Jan Galen Bishop and Steven G Aldana. 1999. Step up to wellness: A stage-based approach. Allyn and Bacon, Boston, MA, USA.

[7] Prakhar Biyani, Cornelia Caragea, Prasenjit Mitra, and John Yen. 2014. Identifying emotional and informational support in online health communities. In Proceedings of COLING 2014, the 25th International Conference on Computational Linguistics: Technical Papers. 827-836.

[8] Katya Borgos-Rodriguez, Kathryn E Ringland, and Anne Marie Piper. 2019. MyAutsomeFamilyLife: Analyzing Parents of Children with Developmental Disabilities on YouTube. Proceedings of the ACM on Human-Computer Interaction 3 , CSCW (2019), 1-26.

[9] Lawrence M Brammer. 1992. Coping with life transitions. International fournal for the Advancement of Counselling 15, 4 (1992), 239-253.

[10] Virginia Braun and Victoria Clarke. 2006. Using thematic analysis in psychology. Qualitative research in psychology 3, 2 (2006), 77-101.

[11] Lauren Britton, Louise Barkhuus, and Bryan Semaan. 2019. "Mothers as Candy Wrappers": Critical Infrastructure Supporting the Transition into Motherhood. Proc. ACM Hum.-Comput. Interact. 3, GROUP, Article 232 (Dec. 2019), 21 pages. https://doi.org/10.1145/3361113

[12] Bradley Carron-Arthur, Kathina Ali, John Alastair Cunningham, and Kathleen Margaret Griffiths. 2015. From help-seekers to influential users: a systematic review of participation styles in online health communities. Fournal of medical Internet research 17, 12 (2015), e271.

[13] Rui EV Carvalho and Carla Teixeira Lopes. 2016. Social network analysis to understand behaviour dynamics in online health communities: A systematic review. In 2016 11th Iberian Conference on Information Systems and Technologies (CISTI). IEEE, 1-7.

[14] Stevie Chancellor, Andrea Hu, and Munmun De Choudhury. 2018. Norms Matter: Contrasting Social Support Around Behavior Change in Online Weight Loss Communities. In Proceedings of the 2018 CHI Conference on Human Factors in Computing Systems (Montreal QC, Canada) (CHI '18). Association for Computing Machinery, New York, NY, USA, 1-14. https://doi.org/10.1145/3173574.3174240

[15] Stevie Chancellor, Zhiyuan Lin, Erica L. Goodman, Stephanie Zerwas, and Munmun De Choudhury. 2016. Quantifying and Predicting Mental Illness Severity in Online Pro-Eating Disorder Communities. In Proceedings of the 19th ACM Conference on Computer-Supported Cooperative Work \& Social Computing (San Francisco, California, USA) (CSCW'16). Association for Computing Machinery, New York, NY, USA, 1171-1184. https://doi.org/10.1145/2818048.2819973

[16] Stevie Chancellor, Jessica Annette Pater, Trustin Clear, Eric Gilbert, and Munmun De Choudhury. 2016. \#thyghgapp: Instagram Content Moderation and Lexical Variation in Pro-Eating Disorder Communities. In Proceedings of the 19th ACM Conference on Computer-Supported Cooperative Work \& Social Computing (San Francisco, California, USA) (CSCW'16). Association for Computing Machinery, New York, NY, USA, 1201-1213. https://doi.org/10.1145/2818048.2819963

[17] Andrea Civan, David W. McDonald, Kenton T. Unruh, and Wanda Pratt. 2009. Locating Patient Expertise in Everyday Life. In Proceedings of the ACM 2009 International Conference on Supporting Group Work (Sanibel Island, Florida, USA) (GROUP '09). Association for Computing Machinery, New York, NY, USA, 291-300. https://doi.org/10.1145/1531674.1531718 
[18] Doğa Corlu, Şeyma Taşel, Semra Gülce Turan, Athanasios Gatos, and Asim Evren Yantaç. 2017. Involving autistics in user experience studies: A critical review. In Proceedings of the 2017 Conference on Designing Interactive Systems. 43-55.

[19] David Denyer and David Tranfield. 2009. Producing a systematic review. (2009).

[20] Halbert L Dunn. 1959. High-level wellness for man and society. American journal of public health and the nations health 49, 6 (1959), 786-792.

[21] Falsh Eurobarometer. 2014. European citizens' digital health literacy. A report to the European Commission (2014).

[22] Gunther Eysenbach, John Powell, Marina Englesakis, Carlos Rizo, and Anita Stern. 2004. Health related virtual communities and electronic support groups: systematic review of the effects of online peer to peer interactions. Bmj 328, 7449 (2004), 1166.

[23] Jessica L Feuston and Anne Marie Piper. 2019. Everyday experiences: small stories and mental illness on Instagram. In Proceedings of the 2019 CHI Conference on Human Factors in Computing Systems. 1-14.

[24] S Fox. 2011. Peer-to-peer healthcare. Pew Internet and American Life Project.

[25] Arthur W Frank. 2016. From sick role to narrative subject: An analytic memoir. Health: 20, 1 (2016), 9-21.

[26] Doğa Gatos and Asim Evren Yantaç. 2020. "Oxygen Mask": Understanding How Autism Parents Seek Support. In Proceedings of the 11th Nordic Conference on Human-Computer Interaction: Shaping Experiences, Shaping Society. 1-11.

[27] Xinning Gui, Yu Chen, Yubo Kou, Katie Pine, and Yunan Chen. 2017. Investigating Support Seeking from Peers for Pregnancy in Online Health Communities. Proc. ACM Hum.-Comput. Interact. 1, CSCW, Article 50 (Dec. 2017), 19 pages. https //doi.org/10.1145/3134685

[28] Asli Günay. 2017. Conceptualization of positive pregnancy experience with the integration of mobile health technologies. Ph.D. Dissertation. Middle East Technical University.

[29] Andrea L. Hartzler, Bridget Weis, Carly Cahill, Wanda Pratt, Albert Park, Uba Backonja, and David W. McDonald. 2016. Design and Usability of Interactive User Profiles for Online Health Communities. ACM Trans. Comput.-Hum. Interact 23, 3, Article 15 (June 2016), 33 pages. https://doi.org/10.1145/2903718

[30] Katrina L. Hinson. 2017. Framing Illness through Facebook Enabled Online Support Groups. Commun. Des. Q. Rev 4, 2b (March 2017), 22-31. https://doi. org/10.1145/3068755.3068758

[31] Sebastian Hökby, Gergö Hadlaczky, Joakim Westerlund, Danuta Wasserman, Judit Balazs, Arunas Germanavicius, Núria Machín, Gergely Meszaros, Marco Sarchiapone, Airi Värnik, et al. 2016. Are mental health effects of internet use attributable to the web-based content or perceived consequences of usage? A longitudinal study of European adolescents. FMIR mental health 3, 3 (2016), e31.

[32] Jina Huh. 2015. Clinical Questions in Online Health Communities: The Case of "See Your Doctor" Threads. In Proceedings of the 18th ACM Conference on Computer Supported Cooperative Work \& Social Computing (Vancouver, BC, Canada) (CSCW '15). Association for Computing Machinery, New York, NY, USA, 1488-1499. https://doi.org/10.1145/2675133.2675259

[33] Jina Huh and Wanda Pratt. 2014. Weaving Clinical Expertise in Online Health Communities. In Proceedings of the SIGCHI Conference on Human Factors in Computing Systems (Toronto, Ontario, Canada) (CHI '14). Association for Computing Machinery, New York, NY, USA, 1355-1364. https://doi.org/10.1145/2556288. 2557293

[34] Khalid S Khan, Regina Kunz, Jos Kleijnen, and Gerd Antes. 2003. Five steps to conducting a systematic review. Journal of the royal society of medicine 96,3 (2003), 118-121

[35] Natasja Kingod, Bryan Cleal, Ayo Wahlberg, and Gitte R Husted. 2017. Online peer-to-peer communities in the daily lives of people with chronic illness: a qualitative systematic review. Qualitative Health Research 27, 1 (2017), 89-99.

[36] Lisa M Kopf, Simone Graetzer, and Jina Huh. 2015. Videos influence behavior change measures for voice and speech in individuals with Parkinson's disease. In Proceedings of the conference on Wireless Health. 1-7.

[37] Annie YS Lau and Trevor MY Kwok. 2009. Social features in online communities for healthcare consumers-a review. In International Conference on Online Communities and Social Computing. Springer, 682-689.

[38] Guo Li, Xiaomu Zhou, Tun Lu, Jiang Yang, and Ning Gu. 2016. SunForum: Under standing Depression in a Chinese Online Community. In Proceedings of the 19th ACM Conference on Computer-Supported Cooperative Work \& Social Computing (San Francisco, California, USA) (CSCW'16). Association for Computing Machinery, New York, NY, USA, 515-526. https://doi.org/10.1145/2818048.2819994

[39] Victor Li, David W. McDonald, Elizabeth V. Eikey, Jessica Sweeney, Janessa Escajeda, Guarav Dubey, Kaitlin Riley, Erika S. Poole, and Eric B. Hekler. 2014. Losing It Online: Characterizing Participation in an Online Weight Loss Community. In Proceedings of the 18th International Conference on Supporting Group Work (Sanibel Island, Florida, USA) (GROUP '14). Association for Computing Machinery, New York, NY, USA, 35-45. https://doi.org/10.1145/2660398.2660416

[40] Thurmon E Lockhart, Adam T Barth, Xiaoyue Zhang, Rahul Songra, Emaad Abdel-Rahman, and John Lach. 2010. Portable, non-invasive fall risk assessment in end stage renal disease patients on hemodialysis. In Wireless Health 2010 84-93.
[41] Haiwei Ma, C. Estelle Smith, Lu He, Saumik Narayanan, Robert A. Giaquinto, Roni Evans, Linda Hanson, and Svetlana Yarosh. 2017. Write for Life: Persisting in Online Health Communities through Expressive Writing and Social Support. Proc. ACM Hum.-Comput. Interact. 1, CSCW, Article 73 (Dec. 2017), 24 pages. https://doi.org/10.1145/3134708

[42] Diana MacLean, Sonal Gupta, Anna Lembke, Christopher Manning, and Jeffrey Heer. 2015. Forum77: An Analysis of an Online Health Forum Dedicated to Addiction Recovery. In Proceedings of the 18th ACM Conference on Computer Supported Cooperative Work \& Social Computing (Vancouver, BC, Canada) (CSCW '15). Association for Computing Machinery, New York, NY, USA, 1511-1526. https://doi.org/10.1145/2675133.2675146

[43] Sanna Malinen. 2015. Understanding user participation in online communities: A systematic literature review of empirical studies. Computers in human behavior 46 (2015), 228-238.

[44] Diane Maloney-Krichmar and Jenny Preece. 2005. A multilevel analysis of sociability, usability, and community dynamics in an online health community. ACM Transactions on Computer-Human Interaction (TOCHI) 12, 2 (2005), 201-232.

[45] Lena Mamykina, Drashko Nakikj, and Noemie Elhadad. 2015. Collective Sensemaking in Online Health Forums. In Proceedings of the 33rd Annual ACM Conference on Human Factors in Computing Systems (Seoul, Republic of Korea) (CHI '15). Association for Computing Machinery, New York, NY, USA, 3217-3226. https://doi.org/10.1145/2702123.2702566

[46] Michael Massimi, Jackie L. Bender, Holly O. Witteman, and Osman H. Ahmed. 2014. Life Transitions and Online Health Communities: Reflecting on Adoption, Use, and Disengagement. In Proceedings of the 17th ACM Conference on Computer Supported Cooperative Work \& Social Computing (Baltimore, Maryland, USA) (CSCW'14). Association for Computing Machinery, New York, NY, USA, 1491-1501. https://doi.org/10.1145/2531602.2531622

[47] Rebecca McKee. 2013. Ethical issues in using social media for health and health care research. Health Policy 110, 2-3 (2013), 298-301.

[48] Gordon D Miller and Leslie T Foster. 2010. Critical synthesis of wellness literature. (2010).

[49] S Anne Moorhead, Diane E Hazlett, Laura Harrison, Jennifer K Carroll, Anthea Irwin, and Ciska Hoving. 2013. A new dimension of health care: systematic review of the uses, benefits, and limitations of social media for health communication. fournal of medical Internet research 15, 4 (2013), e85.

[50] Sean A Munson, Hasan Cavusoglu, Larry Frisch, and Sidney Fels. 2013. Sociotechnical challenges and progress in using social media for health. Fournal of medical Internet research 15,10 (2013), e226.

[51] Drashko Nakikj and Lena Mamykina. 2017. A Park or A Highway: Overcoming Tensions in Designing for Socio-Emotional and Informational Needs in Online Health Communities. In Proceedings of the 2017 ACM Conference on Computer Supported Cooperative Work and Social Computing (Portland, Oregon, USA) (CSCW '17). Association for Computing Machinery, New York, NY, USA, 1304-1319. https://doi.org/10.1145/2998181.2998339

[52] Drashko Nakikj and Lena Mamykina. 2018. Lost in Migration: Information Management and Community Building in an Online Health Community. In Proceedings of the 2018 CHI Conference on Human Factors in Computing Systems (Montreal QC, Canada) (CHI '18). Association for Computing Machinery, New York, NY, USA, 1-14. https://doi.org/10.1145/3173574.3173720

[53] P. Nambisan. 2011. Information seeking and social support in online health communities: impact on patients' perceived empathy. Fournal of the American Medical Informatics Association : JAMIA 183 (2011), 298-304.

[54] Arvind Narayanan and Vitaly Shmatikov. 2010. Myths and fallacies of" personally identifiable information". Commun. ACM 53, 6 (2010), 24-26.

[55] Michael P O’Donnell. 2009. Definition of health promotion 2.0: embracing passion, enhancing motivation, recognizing dynamic balance, and creating opportunities.

[56] Kathleen O'Leary, Arpita Bhattacharya, Sean A. Munson, Jacob O. Wobbrock, and Wanda Pratt. 2017. Design Opportunities for Mental Health Peer Support Technologies. In Proceedings of the 2017 ACM Conference on Computer Supported Cooperative Work and Social Computing (Portland, Oregon, USA) (CSCW '17). Association for Computing Machinery, New York, NY, USA, 1470-1484. https: //doi.org/10.1145/2998181.2998349

[57] Kathleen O'Leary, Stephen M. Schueller, Jacob O. Wobbrock, and Wanda Pratt. 2018. "Suddenly, We Got to Become Therapists for Each Other": Designing Peer Support Chats for Mental Health. In Proceedings of the 2018 CHI Conference on Human Factors in Computing Systems (Montreal QC, Canada) (CHI '18). Association for Computing Machinery, New York, NY, USA, 1-14. https://doi.org/10.1145/3173574.3173905

[58] Michael Oliver, Debora Dr, and Subimal Datta. 2018. Health to Wellness: A Review of Wellness Models and Transitioning Back to Health. 9 (01 2018), 41-56. https://doi.org/10.18848/2156-8960/CGP/v09i01/41-56

[59] World Health Organization et al. 1995. Constitution of the world health organization. (1995)

[60] Zhenhui Peng, Qingyu Guo, Ka Wing Tsang, and Xiaojuan Ma. 2020. Exploring the Effects of Technological Writing Assistance for Support Providers in Online Mental Health Community. In Proceedings of the 2020 CHI Conference on Human Factors in Computing Systems (Honolulu, HI, USA) (CHI '20). Association for 
Computing Machinery, New York, NY, USA, 1-15. https://doi.org/10.1145/ 3313831.3376695

[61] Bernd Ploderer, Wally Smith, Steve Howard, Jon Pearce, and Ron Borland. 2013 Patterns of Support in an Online Community for Smoking Cessation. In Proceedings of the 6th International Conference on Communities and Technologies (Munich, Germany) (C\&T '13). Association for Computing Machinery, New York, NY, USA, 26-35. https://doi.org/10.1145/2482991.2482992

[62] PRISMA. 2015. Prisma Statement. Retrieved September 13, 2020 from http: //prisma-statement.org

[63] Yada Pruksachatkun, Sachin R. Pendse, and Amit Sharma. 2019. Moments of Change: Analyzing Peer-Based Cognitive Support in Online Mental Health Forums. In Proceedings of the 2019 CHI Conference on Human Factors in Computing Systems (Glasgow, Scotland Uk) (CHI '19). Association for Computing Machinery, New York, NY, USA, 1-13. https://doi.org/10.1145/3290605.3300294

[64] Doreen Reifegerste, Katrin Wasgien, and Lutz M Hagen. 2017. Online social support for obese adults: Exploring the role of forum activity. International journal of medical informatics 101 (2017), 1-8.

[65] Pew Research. 2009. The Social Life of Health Information.

[66] Kathryn E Ringland, Christine T Wolf, Lynn Dombrowski, and Gillian R Hayes. 2015. Making" Safe" Community-Centered Practices in a Virtual World Dedicated to Children with Autism. In Proceedings of the 18th ACM Conference on Computer Supported Cooperative Work \& Social Computing. 1788-1800.

[67] Sabirat Rubya and Svetlana Yarosh. 2017. Video-Mediated Peer Support in an Online Community for Recovery from Substance Use Disorders. In Proceedings of the 2017 ACM Conference on Computer Supported Cooperative Work and Social Computing (Portland, Oregon, USA) (CSCW '17). Association for Computing Machinery, New York, NY, USA, 1454-1469. https://doi.org/10.1145/2998181. 2998246

[68] Eva Sharma and Munmun De Choudhury. 2018. Mental Health Support and Its Relationship to Linguistic Accommodation in Online Communities. In Proceedings of the 2018 CHI Conference on Human Factors in Computing Systems (Montreal QC, Canada) (CHI '18). Association for Computing Machinery, New York, NY, USA, 1-13. https://doi.org/10.1145/3173574.3174215

[69] Elizabeth Sillence, Claire Hardy, and Pam Briggs. 2013. Why Don't We Trust Health Websites That Help Us Help Each Other? An Analysis of Online Peer-toPeer Healthcare. In Proceedings of the 5th Annual ACM Web Science Conference (Paris, France) (WebSci '13). Association for Computing Machinery, New York, NY, USA, 396-404. https://doi.org/10.1145/2464464.2464488

[70] Elisabeth Staksrud and Sonia Livingstone. 2009. Children and online risk: Powerless victims or resourceful participants? Information, Communication \& Society 12, 3 (2009), 364-387.

[71] Joanna Taylor and Claudia Pagliari. 2018. Mining social media data: How are research sponsors and researchers addressing the ethical challenges? Research Ethics 14, 2 (2018), 1-39.

[72] Matthieu Tixier, Gérald Gaglio, and Myriam Lewkowicz. 2009. Translating Social Support Practices into Online Services for Family Caregivers. In Proceedings of the ACM 2009 International Conference on Supporting Group Work (Sanibel Island, Florida, USA) (GROUP '09). Association for Computing Machinery, New York, NY, USA, 71-80. https://doi.org/10.1145/1531674.1531685

[73] Matthieu Tixier and Myriam Lewkowicz. 2016. "Counting on the Group": Reconciling Online and Offline Social Support among Older Informal Caregivers. In Proceedings of the 2016 CHI Conference on Human Factors in Computing Systems (San Jose, California, USA) (CHI '16). Association for Computing Machinery, New York, NY, USA, 3545-3558. https://doi.org/10.1145/2858036.2858477

[74] Mies C van Eenbergen, Lonneke V van de Poll-Franse, Peter Heine, and Floortje Mols. 2017. The impact of participation in online cancer communities on patient reported outcomes: systematic review. FMIR cancer 3, 2 (2017), e15.

[75] Tatiana A. Vlahovic, Yi-Chia Wang, Robert E. Kraut, and John M. Levine. 2014 Support Matching and Satisfaction in an Online Breast Cancer Support Community. In Proceedings of the SIGCHI Conference on Human Factors in Computing Systems (Toronto, Ontario, Canada) (CHI '14). Association for Computing Machinery, New York, NY, USA, 1625-1634. https://doi.org/10.1145/2556288.2557108

[76] Xunyi Wang, Srikanth Parameswaran, Darshan Bagul, and Rajiv Kishore. 2017 Does Online Social Support Work in Stigmatized Chronic Diseases? A Study of the Impacts of Different Facets of Informational and Emotional Support on Self-Care Behavior in an HIV Online Forum.. In ICIS.

[77] Xi Wang, Kang Zhao, and Nick Street. 2017. Analyzing and predicting user participations in online health communities: a social support perspective. fournal of medical Internet research 19, 4 (2017), e130.

[78] Chieh-Lin Wu, Bing-Hsun Wu, Yun-Ting Lin, Pengfei Wang, and Yining Zhou 2016. Moments A Wearable Device for Early Stage AD Patients to Maintain Their Autonomy. In Proceedings of the 2016 CHI Conference Extended Abstracts on Human Factors in Computing Systems. 74-79.

[79] Zhijun Yan, Tianmei Wang, Yi Chen, and Han Zhang. 2016. Knowledge sharing in online health communities: A social exchange theory perspective. Information \& Management 53, 5 (2016), 643-653.

[80] Diyi Yang, Robert Kraut, and John M. Levine. 2017. Commitment of Newcomers and Old-Timers to Online Health Support Communities. In Proceedings of the 2017
CHI Conference on Human Factors in Computing Systems (Denver, Colorado, USA) (CHI '17). Association for Computing Machinery, New York, NY, USA, 6363-6375. https://doi.org/10.1145/3025453.3026008

[81] Diyi Yang Robert E. Kraut, Tenbroeck Smith, Elijah Mayfield, and Dan Jurafsky. 2019. Seekers, Providers, Welcomers, and Storytellers: Modeling Social Roles in Online Health Communities. In Proceedings of the 2019 CHI Conference on Human Factors in Computing Systems (Glasgow, Scotland Uk) (CHI '19). Association for Computing Machinery, New York, NY, USA, 1-14. https://doi.org/10.1145/ 3290605.3300574

[82] Diyi Yang, Zheng Yao, Joseph Seering, and Robert Kraut. 2019. The Channel Matters: Self-Disclosure, Reciprocity and Social Support in Online Cancer Support Groups. In Proceedings of the 2019 CHI Conference on Human Factors in Computing Systems (Glasgow, Scotland Uk) (CHI '19). Association for Computing Machinery, New York, NY, USA, 1-15. https://doi.org/10.1145/3290605.3300261

[83] Zeynep Yildiz, Doğa Gatos, Özge Subasi, Asim Evren Yantac, and Kemal Kuscu. 2019. Examining Online Practices of an Autism Parent Community in Turkey: Goals, Needs, and Opportunities. In Proceedings of the 2019 on Designing Interactive Systems Conference (San Diego, CA, USA) (DIS '19). Association for Computing Machinery, New York, NY, USA, 373-384. https://doi.org/10.1145/ 3322276.3322344

[84] Alyson L. Young and Andrew D. Miller. 2019. "This Girl is on Fire": Sensemaking in an Online Health Community for Vulvodynia. In Proceedings of the 2019 CHI Conference on Human Factors in Computing Systems (Glasgow, Scotland Uk) (CHI '19). Association for Computing Machinery, New York, NY, USA, 1-13. https://doi.org/10.1145/3290605.3300359

[85] Shaodian Zhang, Erin O'Carroll Bantum, Jason Owen, Suzanne Bakken, and Noémie Elhadad. 2017. Online cancer communities as informatics intervention for social support: conceptualization, characterization, and impact. fournal of the American Medical Informatics Association 24, 2 (2017), 451-459.

[86] Tao Zhou. 2019. Examining users' knowledge sharing behaviour in online health communities. Data Technologies and Applications (2019).

[87] Xiaomu Zhou, Si Sun, and Jiang Yang. 2014. Sweet Home: Understanding Diabetes Management via a Chinese Online Community. In Proceedings of the SIGCHI Conference on Human Factors in Computing Systems (Toronto, Ontario, Canada) (CHI '14). Association for Computing Machinery, New York, NY, USA, 3997-4006. https://doi.org/10.1145/2556288.2557344 